\title{
Community-Based Rapid HIV Testing in Brazil for Vulnerable Populations: Whom are We Reaching?
}

Clarissa Habckost Dutra de Barros ${ }^{1}$, Meritxell Sabidó ${ }^{1,2}$, Tainah Dourado de Miranda Lobo ${ }^{1}$, Roberta Pati Pascom ${ }^{1}$, Elisiane Pasini ${ }^{1}$, Fábio Caldas de Mesquita ${ }^{3}$ and Schwartz Benzaken ${ }^{1,4 *}$

${ }^{1}$ Department of STI, AIDS and Viral Hepatitis, Secretary for Health Surveillance, Ministry of Health Brazil, Brasilia, DF, Brazil

${ }^{2}$ TransLab Department of Medical Sciences, University of Girona, Catalonia. CIBER Epidemiology and Public Health, CIBERESP, Spain

${ }^{3}$ Faculdade de Medicina da Universidade de São Paulo - FM/USP São Paulo/SP, Brazil

${ }^{4}$ Tropical Medicine Foundation Dr. Heitor Vieira Dourado (FMT-HVD) (FMT-HVD), Manaus, Amazonas State, Brazil

\begin{abstract}
Objective: Rapid HIV testing and counselling in community settings delivered by trained peers can allow easier access to and uptake of HIV testing and care. The programme 'Live Better Knowing' offers rapid HIV testing to key populations across Brazil. This cross-sectional study aims at describing the HIV prevalence in population subgroups and at assessing the association of sex work (SW) and/or drug use with a positive HIV result.

Methods: Between January 2013 and April 2016, key populations were invited for HIV rapid oral fluid tests (DPP HIV$1 / 2$ Bio-Manguinhos/Fiocruz, Rio de Janeiro, Brazil) by peers. HIV testing was offered in identified venues and areas where these populations socialize. Participants were interviewed using a brief questionnaire about their socio-demographic and risk behaviour. Multivariable logistic regression was used to assess the effect of SW and/or drug use on the risk of HIV.

Results: Among the 43,358 participants, $42.9 \%$ were ciswomen, $2.9 \%$ were transvestites, $2.7 \%$ were transgender, $19.8 \%$ were men who have sex with men (MSM) and $31.9 \%$ were heterosexual men. Overall, $52.1 \%$ were first-time HIV testers. The HIV prevalence among the ciswomen, transvestites, transgender people, MSM and heterosexual men was $0.9 \%, 7.2 \%, 4 \%, 3.4 \%$ and $1 \%$, respectively. Individuals who performed SW and used drugs had the highest HIV prevalence within each population group (ciswomen $1.7 \%$, transvestites $8.1 \%$, transgender $7.4 \%$, MSM $5.9 \%$ and heterosexual men $2.6 \%)$. The adjusted odds ratios with $95 \%$ confidence intervals for selling sex combined with drug use were 2.90 (1.21-6.96) in ciswomen, 25.01 (2.67-234.57) in transgender people and 2.42 (1.31-4.48) in MSM.
\end{abstract}

Conclusion: This outreach programme attracted persons with high risk practices who had never been tested. Our findings suggest that transvestites and transgender people (particularly those who sell sex) constitute a high risk population for HIV in urgent need of enhanced prevention, treatment and care services.

Keywords: Combination HIV prevention; HIV screening; Oral fluid; Vulnerable populations; Brazil

\section{Introduction}

Brazil's HIV epidemic continues to have profound effect on key populations [1]. Adult HIV prevalence is $0.4 \%$ [2]. HIV infection rates are disproportionately higher in men who have sex with men (MSM; $14.2 \%)$ [3], transgender women [4], individuals engaged in sex work (SW; 4.8\%) and intravenous drug users (IDUs; 5.9\%) [5,6]. Overall, 43.2\% of HIV infections in males in 2013 were attributed to sex between men [7]. This high burden of infection indicates significant exposure and is coupled with an unmet need for enhanced prevention, treatment, and care services [1]. Rates of HIV testing in key populations are low, with only $18 \%$ of sex workers and $19.1 \%$ of MSM reporting being tested for HIV in the last 12 months knowing their results [2]. In a national sample of MSM, only $30 \%$ reported consistent condom use during the last year [8].

In Brazil, voluntary counselling and testing (VCT) for HIV is offered by publicly funded testing and counselling services. Over the past few years, actions to strengthen early HIV diagnosis have been taken and have increased distribution of rapid HIV testing and expanded the number of facilities providing VCT for HIV. However, HIV testing is still offered largely at clinical sites, and outreach programmes remain scarce [1]. This situation results in missed HIV prevention opportunities, particularly for most at-risk populations who might not seek testing in traditional facility-based settings $[9,10]$.

Community-based testing approaches have increased testing uptake among key populations [11] and can facilitate linkage to care
[12]. Importantly, non-invasive options for specimen collection, such as saliva-based tests, can facilitate access to and acceptability of HIV testing [13]. Peer outreach activities have demonstrated to be effective in some countries, and income levels have increased the usage of HIV testing among key populations, such as MSM and individuals engaged in SW. Moreover, HIV testing can reach hard-to-reach populations that previously have not had access [14-16].

In light of the country's epidemiological scenario and of unmet needs for prevention, the Department of STI, AIDS and Viral Hepatitis (DDAHV) of the Brazilian Ministry of Health launched an intervention in 2013 called "Viva Melhor Sabendo" (Live better knowing). This programme involved a combination of HIV prevention and intervention strategies targeted at the most at-risk groups with measures to achieve a synergistic effect in reducing HIV transmission [17]. The components of the intervention were previously described in detail [18]. Briefly, the programme consists of an outreach programme that offers rapid oral

${ }^{*}$ Corresponding author: Adele Schwartz Benzaken, Tropical Medicine Foundation Dr. Heitor Vieira Dourado (FMT-HVD) (FMT-HVD), Manaus, Amazonas State Brazil, Tel: 5561 3315-7603; E-mail: adele.benzaken@aids.gov.br

Received March 10, 2017; Accepted March 17, 2017; Published March 24, 2017

Citation: de Barros CHD, Sabidó M, de Miranda Lobo TD, Pascom RP, Pasini E, et al. (2017) Community-Based Rapid HIV Testing in Brazil for Vulnerable Populations: Whom are We Reaching? J AIDS Clin Res 8: 679. doi: 10.4172/2155-6113.1000679

Copyright: (C) 2017 de Barros CHD, et al. This is an open-access article distributed under the terms of the Creative Commons Attribution License, which permits unrestricted use, distribution, and reproduction in any medium, provided the original author and source are credited. 
fluid HIV testing by peers at community level. DDAHV collaborates with civil society organizations closely linked to key populations, which is crucial for the design and implementation of the whole intervention and allows those diagnosed with HIV to be connected with the health system.

Our aim was to describe the prevalence of HIV in subgroups of key populations targeted by the intervention 'Live Better Knowing' and to assess the association of SW and/or drug use with a positive HIV result. In the context of nationwide public health care system, it is extremely important to understand the extent of the HIV burden in order to implement targeted and evidence-based measures that address groups most affected by the HIV epidemic and those most at-risk for disease acquisition and transmission.

\section{Methods}

\section{Study design}

A cross-sectional study was implemented using data collected during peer community-based HIV testing intervention performed in 38 Brazilian cities distributed across the five country regions.

The strategy was managed by DDAHV. Forty local nongovernmental organizations (NGOs) were invited to participate (10 dedicated to MSM, 10 to transgender persons, 10 to SW and 10 to drug users) based on their community experience and proximity to the targeted populations. Of those organizations, four were excluded for administrative or financial issues and 34 accepted participation. Since July of 2014, 76 additional NGOs have joined by responding to a call for expression of interest, resulting in the participation of 110 NGOs.

NGOs staff were trained in counselling, risk assessment, performance of rapid HIV oral fluid test, reading and delivering results, and patient referral flow. Emphasis was given to confidentiality, respect and the Brazilian human rights framework on HIV.

NGOs staff were also trained on how to implement and monitor the intervention. Detailed information was provided in an institutional guide booklet. A video on oral fluid HIV testing was developed to increase the uniformity of testing performance. The initial implementation was also supervised and monitored by DDAHV staff [19].

\section{Sampling}

To establish the operational framework of the intervention, community social mapping was conducted to identify and locate commercial sex venues, nooks for drug consumption, and gay scene in each city. Squares, bars, discos, hotels and motels were located based on direct observation of daily activities and interviews with key informants from the community. Those sites were also visited to obtain in-depth information.

These maps were used to guide the selection of intervention sites. The target populations were informed of the intervention aims during advertising campaigns (flyers, ads, talks offered by peers and radio for small cities) at commercial and non-commercial venues as well as via NGOs, gay websites, and magazines.

Between January 2013 and April 2016, consecutive subjects from key populations (i.e., sex workers, drug users, MSM and transgender people) and young people aged 15-24 years at high risk for acquiring $\mathrm{HIV}$ at the testing sites were invited to participate in the intervention. Nevertheless, anyone showing interest in being screened for HIV could participate.

\section{Study population}

Subjects were eligible for interview if they agreed to provide oral fluid sample and signed an informed consent form. We defined individuals engage $\mathrm{d}$ in SW as women or men who self-identified as sex workers. Transgender people were defined as those who selfidentified as such or whose gender identity differed from their birth sex. Transvestites were those with a male birth sex and female gender identity. In Brazil, transgender women and transvestites both have a male birth sex and female social expression. However, they differ in their political and/or subjective self-identification. Ciswomen were those who had a female biological sex and female gender identity. The MSM group included men who self-identified as homosexuals or who reported having any type of sex (anal, oral or other non-penetrative sex) at least once with another man. Individuals who declared use of at least one psychoactive substance regardless of administration route were considered drug users.

\section{Counseling and testing}

The approach and recruitment procedures were similar in all settings. After obtaining written informed consent from the participants, trained peer staff conducted pre-test HIV orientation. The peer interviewed the participants using a structured questionnaire including sociodemographic characteristics, sexual practices, condom use at last sexual intercourse, sharing drug or injecting equipment, drug use, STI (sexually transmitted infection) symptoms during last 12 months and HIV testing history. As part of the intervention, female and male condoms and lubricant gel were distributed.

Oral fluid samples were collected by peer staff using HIV rapid oral fluid assay (DPP HIV-1/2 Bio-Manguinhos; Fiocruz, Rio de Janeiro, Brazil) (sensitivity: 98.9-100\%; specificity: 99.9-100\%) [20]. Participants received their results within $30 \mathrm{~min}$. Participants with a reactive HIV rapid test result received post-test counselling and were referred to the corresponding HIV service for HIV diagnosis and enrolment in care. NGO teams were responsible for following up with the people who both tested positive for HIV and agreed to follow-up prior to their linkage to care.

\section{Data Analysis}

Data were analysed using StataV11.0(StataCorp LP, College Station, TX, USA) and described using frequencies, medians and interquartile ranges. Conversely, data were described using percentages and means with standard deviations (SD) when appropriate. Psychoactive substance consumption was further categorized by number of psychoactive substances used regardless of whether the substance was injectable.

For the purpose of this study, the subjects were classified according to the following groups: ciswomen, transvestites, transgender women, transgender men, MSM and heterosexual men. To classify participants according to their vulnerability to HIV infection, we generated an indicator that stratified these groups into four categories based on SW and/or drug use as follows: 'no SW or drug use', 'only SW', 'only drug use' and 'SW and drug use'. The HIV prevalence was calculated as the proportion of participants with a reactive HIV test result among those tested.

Univariable and multivariable analyses using logistic regression were conducted to assess the effect of SW and/or drug use on the HIV rate. Five different models were built (one for each population group). Crude and adjusted odds ratios (AOR) with 95\% CIs were used as measures of 
Citation: de Barros CHD, Sabidó M, de Miranda Lobo TD, Pascom RP, Pasini E, et al. (2017) Community-Based Rapid HIV Testing in Brazil for Vulnerable Populations: Whom are We Reaching? J AIDS Clin Res 8: 679. doi: 10.4172/2155-6113.1000679

Page 3 of 7

association. The independent variables used for the adjustment were age, race/colour, education level, condom use at last sexual intercourse, presence of STI symptoms during last 12 months, needle or drug equipment sharing, first time HIV testing, and number of psychoactive drugs used. The level of significance was established at a $\mathrm{P}$ value $<0.05$.

\section{Ethics}

The intervention Live Better Knowing was part of the Ministry of Health strategic plan to enhance HIV testing and was not assessed by an ethical committee board. All participants were informed about HIV VCT activities and signed a consent form prior to testing. The questionnaire and oral fluid sample identification was optional. In Brazil, according to law CFM n ${ }^{\circ} 1.865 / 96$, authorization or consent from parents or responsible person is not needed to perform an HIV test in individuals between 12 and 18 years of age if the test is voluntary and consent is provided by the adolescent, provided that the adolescent has the capacity to evaluate their problem and take actions accordingly.

\section{Results}

\section{Sample characteristics}

Overall, 43,358 subjects participated in the study, of which $42.9 \%$ were ciswomen, $2.9 \%$ were transvestites, $2.7 \%$ were transgender $19.8 \%$ were MSM and $31.9 \%$ were heterosexual men. Table 1 shows the distribution and characteristics of the participants by population group. The mean age was 29.1 years (SD: 12.2). Regarding education, $28.1 \%$ of the sample had not completed primary school; this proportion was higher among transvestites (32.9\%) and heterosexual men (34\%). Overall, $65.2 \%$ of the sample self-declared as black. Although most (61.5\%) individuals only used drugs, this percentage was higher among

\begin{tabular}{|c|c|c|c|c|c|c|c|c|c|}
\hline & \multirow{2}{*}{\multicolumn{2}{|c|}{$\begin{array}{l}\text { Ciswomen } \\
(\mathrm{N}=18356)\end{array}$}} & \multirow{2}{*}{\multicolumn{2}{|c|}{$\begin{array}{l}\text { Transvestites } \\
\qquad(\mathrm{N}=1232)\end{array}$}} & \multirow{2}{*}{\multicolumn{2}{|c|}{$\begin{array}{l}\text { Transgender } \\
(\mathrm{N}=1148)\end{array}$}} & MSM & & Heterose \\
\hline & & & & & & & \multicolumn{2}{|c|}{$(\mathrm{N}=8519)$} & $(N=1$ \\
\hline & $\mathbf{n}$ & $\%$ & $\mathbf{n}$ & $\%$ & $\mathbf{n}$ & $\%$ & $\mathbf{n}$ & $\%$ & $\mathbf{n}$ \\
\hline \multicolumn{10}{|l|}{ Age, years $(n=42383)$} \\
\hline Mean, SD & \multicolumn{2}{|c|}{$29.5(11.0)$} & \multicolumn{2}{|c|}{$29.3(10.2)$} & \multicolumn{2}{|c|}{$27.5(10.2)$} & \multicolumn{2}{|c|}{$27.8(10.2)$} & 3 \\
\hline $15-24$ & 8433 & 47.1 & 503 & 41.3 & 554 & 49.4 & 3911 & 46.7 & 6113 \\
\hline$>24$ & 9456 & 52.9 & 714 & 58.7 & 568 & 50.6 & 4473 & 53.3 & 7283 \\
\hline \multicolumn{10}{|l|}{ Education $(n=43354)$} \\
\hline Incomplete primary & 4925 & 26.8 & 405 & 32.9 & 302 & 26.3 & 1709 & 20.1 & 4668 \\
\hline Complete primary & 1958 & 10.7 & 170 & 13.8 & 128 & 11.2 & 701 & 8.2 & 1348 \\
\hline High school or more & 11471 & 62.5 & 657 & 53.3 & 718 & 62.5 & 6109 & 71.7 & 7700 \\
\hline \multicolumn{10}{|l|}{ Race/colour $(n=43144)$} \\
\hline White & 6177 & 33.8 & 382 & 31 & 420 & 36.1 & 2652 & 31.5 & 3975 \\
\hline Black $^{*}$ & 11514 & 63.0 & 815 & 66.2 & 687 & 60.1 & 5533 & 65.6 & 9259 \\
\hline Asian & 339 & 1.9 & 22 & 1.8 & 22 & 1.9 & 112 & 1.3 & 192 \\
\hline Indigenous & 259 & 1.4 & 13 & 1.1 & 15 & 1.3 & 135 & 1.6 & 237 \\
\hline
\end{tabular}

\section{SW and drug use $(n=43358)$}

\begin{tabular}{|l|c|c|c|c|c|c|c|c|c|}
\hline No SW nor drug use & 4147 & 22.6 & 33 & 2.7 & 140 & 12.2 & 1357 & 15.9 & 2462 \\
\hline Drug use only & 8907 & 48.5 & 218 & 17.7 & 431 & 37.5 & 6105 & 71.7 & 10804 \\
\hline SW only & 783 & 4.3 & 155 & 12.6 & 89 & 7.8 & 125 & 1.5 & 35 \\
\hline SW and drug use & 4519 & 24.6 & 826 & 67.1 & 488 & 42.5 & 932 & 10.9 & 46 \\
\hline
\end{tabular}

Had any STI symptom during the last 12 months $(n=43358)$

\begin{tabular}{|l|l|l|l|l|l|l|l|l|l|l|l|}
\hline Yes & 1934 & 10.5 & 130 & 10.6 & 123 & 10.7 & 961 & 11.3 \\
\hline
\end{tabular}

Condom use at last sexual intercourse $(n=43358)$

\begin{tabular}{|l|l|l|l|l|l|l|l|l|l|}
\hline Yes & 9165 & 49.9 & 870 & 70.6 & 695 & 60.5 & 4692 & 55.1 & 6278 \\
\hline
\end{tabular}

Shared drug preparation or injecting equipment $(n=43358)$

\begin{tabular}{|l|l|l|l|l|l|l|l|l|l|l|}
\hline Yes & 1053 & 5.7 & 191 & 15.5 & 108 & 9.4 & 784 & 9.2 & 1273 \\
\hline
\end{tabular}

Use of psychoactive substances $(n=43358)$

\begin{tabular}{|c|c|c|c|c|c|c|c|c|c|}
\hline None & 4930 & 26.7 & 188 & 15.3 & 229 & 19.5 & 1482 & 17.4 & 2497 \\
\hline One & 8355 & 45.5 & 377 & 30.6 & 462 & 40.2 & 3608 & 42.4 & 5662 \\
\hline Two & 3246 & 17.7 & 419 & 34 & 265 & 23.1 & 1920 & 22.5 & 3004 \\
\hline Three & 1191 & 6.5 & 169 & 13.7 & 107 & 9.3 & 941 & 11.1 & 1683 \\
\hline Four or more & 634 & 3.5 & 79 & 6.4 & 85 & 7.4 & 568 & 6.7 & 871 \\
\hline
\end{tabular}

Ever tested for HIV $(n=43358)$

\begin{tabular}{|l|l|l|l|l|l|l|l|l|l|l|}
\hline No & 8920 & 48.6 & 414 & 33.6 & 453 & 39.5 & 3787 & 44.4
\end{tabular}

HIV prevalence $(n=43337)$

\begin{tabular}{|l|l|l|l|l|l|l|l|l|l|l|}
\hline Positive & 163 & 0.9 & 88 & 7.2 & 46 & 4 & 290 & 3.4 \\
\hline
\end{tabular}

*Black includes black people and brown (pardos)

Table 1: Main characteristics of participants in 'Live Better Knowing' by population group, Brazil, January. 
Citation: de Barros CHD, Sabidó M, de Miranda Lobo TD, Pascom RP, Pasini E, et al. (2017) Community-Based Rapid HIV Testing in Brazil for Vulnerable Populations: Whom are We Reaching? J AIDS Clin Res 8: 679. doi: 10.4172/2155-6113.1000679

Page 4 of 7

MSM (71.7\%) and heterosexual cismen (78.8\%). In contrast, the percentages of transvestites, transgender and ciswomen who reported only SW $(12.6 \%, 7.8 \%$ and $4.3 \%$, respectively) and SW combined with drug use $(67.1 \%, 47.1 \%$ and $24.6 \%$, respectively) was higher than the percentages in MSM and heterosexual men, respectively.

Half of the sample (50.5\%) reported condom use during last sexual intercourse; this percentage was much higher among transvestites $(70.6 \%)$. In the previous 12 months, $9.1 \%$ of the sample had STI symptoms.
Approximately half of the sample (52.1\%) had never been tested for HIV; heterosexual men group reported the highest first-time HIV testing percentage (66.8\%). Among the participants, $7.9 \%$ had shared drugs or injecting equipment and $5.2 \%$ had used 4 or more psychoactive substances.

\section{Characteristics of the study groups based on SW and drug use behavior}

Figure 1 presents histograms stratified by presence of SW and/or
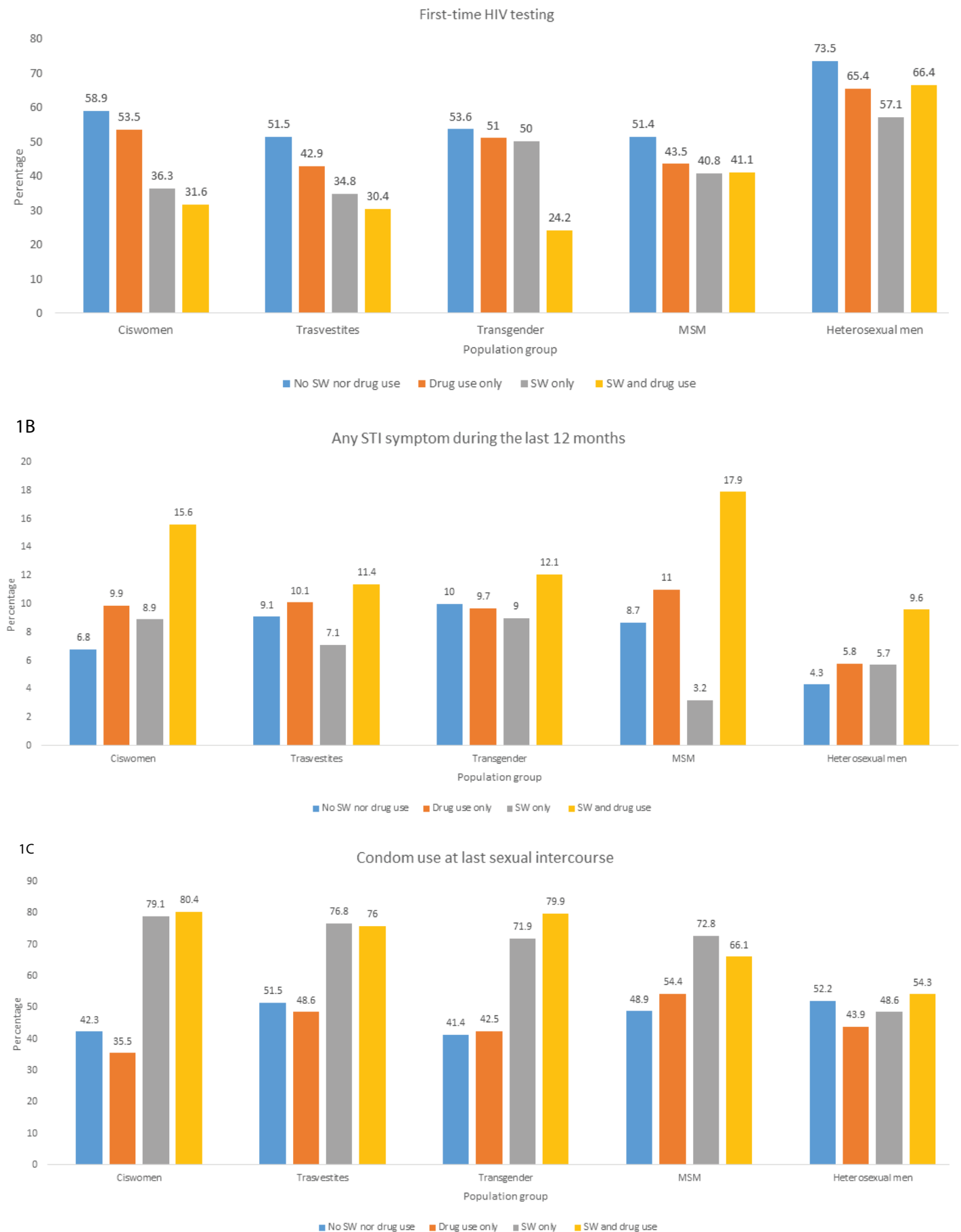

Figure 1: A: Proportion of first-time HIV testing by population group and drug use and/or sex work. B: Proportion of people that had any STI symptoms in the previous 12 months by population group and drug use and/or sex work. C: Proportion of people who reported condom use at last sexual intercourse by population group and drug use and/or sex work. 
drug use behaviour. Within each population group, the individuals with no SW or drug use had the highest percentage of first-time HIV testers (Figure 1A). In contrast, heterosexual men exhibited the lowest percentage of any STI symptom in the last 12 months and within each population group, whereas those who practiced SW and reported drug use presented the highest percentage (Figure 1B). Condom use at last sexual intercourse was similar across population groups, although participants who practiced SW and participants who practiced SW and reported drug use showed a higher percentage of use than those who did not practice SW or use drugs and those who only used drugs (Figure 1C).

\section{HIV prevalence}

Table 1 shows the prevalence of HIV infection based on the oral fluid analysis by population group and Table 2 shows the prevalence of HIV by population group stratified by SW and/or drug use. Transvestites showed the highest prevalence of HIV (7.2\%), followed by transgender people (4\%) and MSM (3.4\%) (Table 1). When results were stratified by SW and/or drug use, HIV prevalence was higher among who only sold sex compared to among participants who only used drugs in ciswoman ( $1.2 \%$ and $0.7 \%$, respectively), transvestite ( $7.1 \%$ and $3.4 \%$, respectively) and transgender subgroups ( $2.3 \%$ and $1.6 \%$, respectively). Within each group, individuals who practiced SW and used drugs had the highest HIV prevalence.

\section{Association of SW and/or drug use behavior with the HIV prevalence}

In the univariate analysis, practicing only SW was associated with having high risk of a positive HIV result among ciswomen. However, this association did not remain in the multivariable model adjusted by age, race/colour, education level, any STI symptom in the last 12 months, condom use during the last sexual intercourse, first-time HIV testing, sharing drug preparation or injecting equipment, and use of psychoactive substances. Among ciswoman, transgender and MSM subgroups, participants who practiced SW and used drugs had a significantly higher chance of being HIV positive in both univariate and multivariate models.

\section{Discussion}

This outreach programme offering rapid HIV testing primarily to key populations was able to attract persons with high risk practices who had never been tested. Our findings suggest that transvestites and transgender people (particularly sex workers) are a population at high risk for HIV and are in urgent need of prevention, treatment and care services.

Our findings confirm significant differences in HIV prevalence between exposure groups, which contribute to our understanding of the concentrated HIV epidemic in Brazil. Transvestites followed by transgender people showed a high burden of HIV infection. Although these two social groups have long been known to be at high risk for HIV acquisition and transmission, they are not included in the Brazilian HIV surveillance system. In both groups, HIV prevalence was higher among those engaging in SW regardless of drug use compared to ciswomen, MSM or cismen who engaged in SW with or without drug use. Sex work has been consistently associated with high risk of HIV acquisition in transgender people [21].

Based on the reporting of SW and drug use, ciswomen, transvestites,

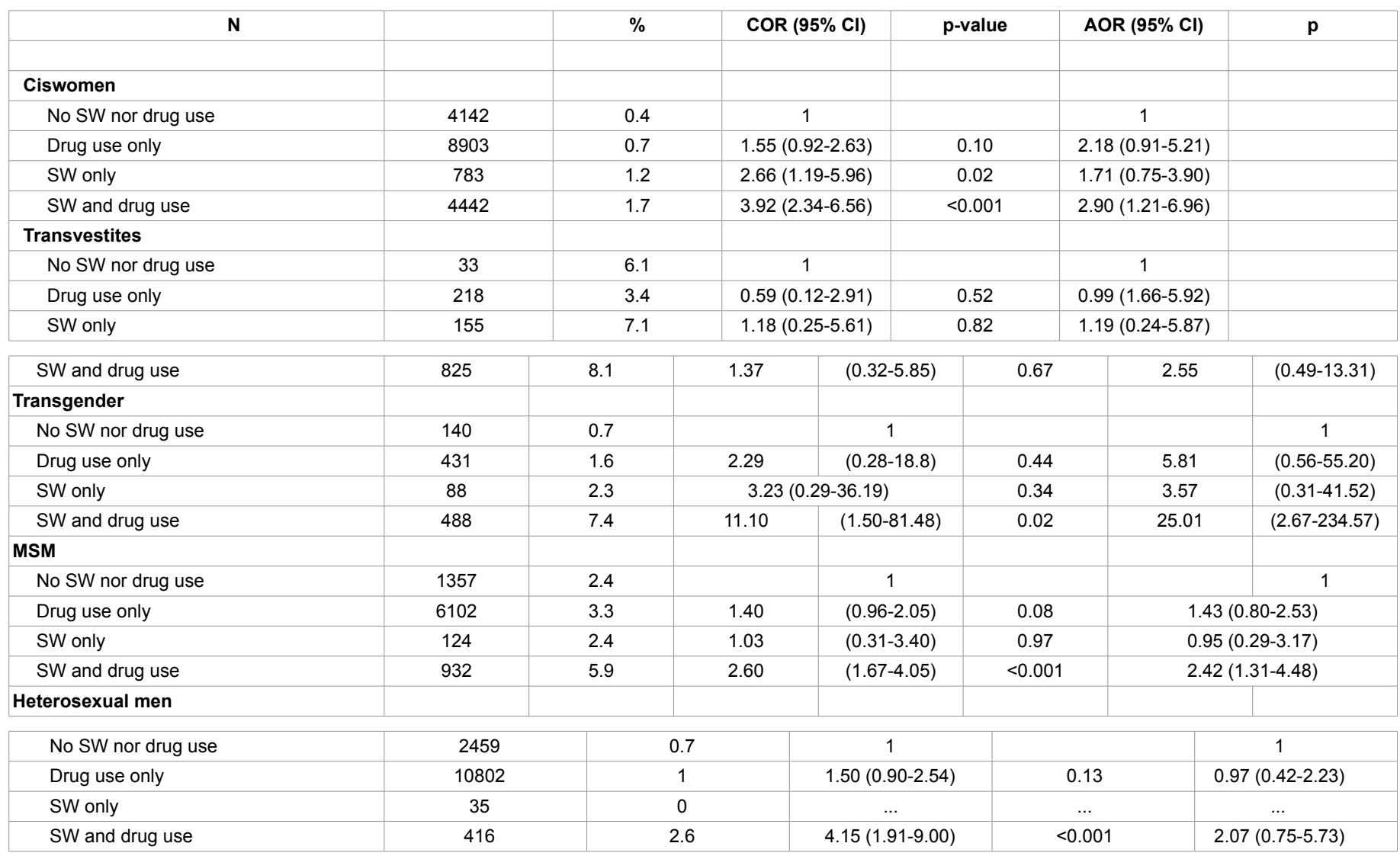

Table 2: Prevalence of HIV and crude and adjusted models predicting HIV infection by population group and/or drug use, Brazil January $2014-$ March 2016. 
and MSM who sold sex and used drugs were more than two times more likely to be living with HIV than those who did not sell sex or consume drugs. In transgender people, the OR of being HIV positive was 25 in those who sold sex and used drugs, which highlighted the disproportionate risk of HIV infection in transgender people practicing these risky behaviours [22].

Brazil's HIV epidemic is predominantly in men, with the highest rate of HIV infection among MSM. Except for the southern region of the country, where high rates of HIV infection have been reported among injection drugs users [1]. This finding contrasts with the low prevalence of HIV found among MSM in our study but may contribute to our understanding of the very high rates of HIV infection in transgender people. Transgender people are often included as a subpopulation of MSM in epidemiological studies because many share risk behaviours with MSMs. However, this classification denies their own identity process. Studies in Brazil often exclude transgender people [3].

In our study, we were not able to separate transgender men and women because we only obtained data on 367 transgender men. Therefore, we analysed the data on transgender people overall. Transgender men have not traditionally been considered at risk for HIV, and their HIV prevalence (1.1\%) was much lower than the prevalence in transgender women (5.4\%) in our study. However, 42 (32.3\%) of the 130 newly diagnosed HIV cases among transgender people occurred in transgender men. In a US study, HIV infection rates were similar for transgender men (10\%) and transgender women (11\%) [23].

Our results revealed that almost half of the persons screened by the programme were undergoing testing for the first time, consistent with findings reported by non-targeted outreach programmes [24,25]. In our sample, transvestites and transgender people were identified as the population groups that were previously tested in the largest proportion. This finding is contrary to results from other studies in which MSM account for the highest proportion of individuals never tested for HIV $[26,27]$. We found differences between the exposure categories in the proportion of first-time testers. Participants who engaged in SW and drug use showed a higher proportion of previous HIV testing, suggesting that individuals exposed to more at-risk behaviours for HIV transmission were more likely to undergo HIV testing.

Similarly, only half of the participants reported condom use during last sexual intercourse, although we found remarkable differences between SW and/or drug use exposure groups. Except for heterosexual men, individuals who sold sex regardless of drug use reported high condom use during last sexual intercourse ( $>70 \%)$. Consistent condom use with appropriate lubricants is an essential prevention method for anyone engaging in anal sex [28]. In Brazil, only 55\% of subjects aged 15-64 years consistently used condoms during the last 12 months [29]. Acceptance and condom use remain challenging even where cost and access might not be barriers. Female condom could be an important alternative, and Brazil has been investing in promoting access, particularly for women who participate in SW.

The present study has several limitations. All testing sites were convenience samples, which might lead to selection bias and compromise the generalizability of the results. Secondly, a potential recall bias or underreporting of some sexual and social behaviours could have occurred. Finally, the cross-sectional design did not permit drawing of causal or temporal inferences. Despite these shortcomings, the present study contributes to improving our understanding of the HIV epidemic in Brazil and has implications for ongoing prevention efforts.
In conclusion, this outreach programme provided rapid HIV testing and counselling services to of individuals who continue to face obstacles in accessing HIV prevention, treatment, and care services. Notably, half of those engaging in testing were first time testers. In view of the high disease burden, we have shown that the current surveillance and prevention interventions for transvestites and transgender people (particularly those who sell sex) clearly need to be enhanced. VCT needs to be more accessible. This approach may be particularly important for transvestites and transgender people, whose vulnerable conditions lead them to a greater risk of stigma, discrimination, and gender-based violence [4]. In 2014, Brazil began observational pilot studies to follow up the provision of oral preexposure prophylaxis (PrEP), which targets all key populations [30]. Given the coexistence of drug use with sex work, comprehensive harm reduction services need to be in place where drug use occurs. Finally, structural policy approaches to change programmes will also be essential for improved universal access to competent clinical prevention, treatment and care services.

\section{Research Highlights (Key Messages)}

A community-based intervention to offer rapid oral HIV testing in Brazil reached vulnerable populations who had never been tested.

Peers were able to perform a rapid HIV test offered within a comprehensive prevention approach tailored to the participants' routines.

Transvestites and transgender people (particularly those who sell sex) are a population at very high risk for HIV infection.

\section{Strengths and Limitations of this Study}

- High acceptability of HIV testing by peers Innovative use of oral fluid tests.

- Partnership between the federal, state and municipality governments and NGOs Strategy tailored for HIV key populations.

- Testing administrated outside of traditional health facilities all testing sites were convenience samples.

- Recall bias or underreporting of some sexual and social behaviours cross-sectional design did not permit drawing of causal or temporal inferences.

- Despite these shortcomings, the present study contributes to improving our understanding of the HIV epidemic in Brazil and has implications for ongoing prevention efforts.

\section{Data Sharing Statement}

The strategy described in this study is being expanded; therefore, there are updated data from new tests and questionnaires that are being performed. Furthermore, not all data available on the questionnaires was used to write this paper. Extra data is available by e-mailing: adele. benzaken@aids.gov.br

\section{Financial Support}

Financial support for this programme was provided by the Department of STI, AIDS and Viral Hepatitis, Secretary for Health Surveillance, Ministry of Health Brazil. 
Citation: de Barros CHD, Sabidó M, de Miranda Lobo TD, Pascom RP, Pasini E, et al. (2017) Community-Based Rapid HIV Testing in Brazil for Vulnerable Populations: Whom are We Reaching? J AIDS Clin Res 8: 679. doi: 10.4172/2155-6113.1000679

\section{Acknowledgement}

We thank the non-governmental organizations, government officials and experts in the programme areas. This programme was funded by the Department of STI, AIDS and Viral Hepatitis of the Brazilian Ministry of Health.

\section{Contributorship Statement}

FM and ASB provided institutional support and coordinated the study. ARP and CHDB designed the study and TL and EP are part of the team responsible for fieldwork supervision. MS analysed data and wrote the manuscript. All of the authors interpreted the data, critically revised the manuscript for important intellectual content and gave final approval for this version to be published.

\section{References}

1. Department of Sexually Transmitted Infections, AIDS and Viral Hepatitis. Secretariat of Health Surveillance, Brazil Ministry of Health (2015) The Brazilian response to HIV and AIDS Global AIDS. Response progress reporting narrative report.

2. Department of Sexually Transmitted Infections, AIDS and Viral Hepatitis, Secretariat of Health Surveillance, Brazil Ministry of Health (2014) Global AIDS Response progress reporting narrative report.

3. Kerr LR, Mota RS, Kendall C, Pinho A, Mello MB, et al. (2012) HIV among MSM in a large middle-income country. AIDS 27: 427-435.

4. Baral SD, Poteat T, Stromdahl S, Wirtz AL, Guadamuz TE, et al. (2013) Worldwide burden of HIV in transgender women: A systematic review and meta-analysis. Lancet Infect Dis 13: 214-222.

5. Szwarcwald CL, de Souza Junior PR, Damacena GN, et al. (2011) Analysis of data collected by RDS among sex workers in 10 Brazilian cities, 2009 Estimation of the prevalence of HIV, variance and design effect. J Acquir Immune Defic Syndr 57: S129-S135.

6. Bastos $\mathrm{FI}$ (2009) Rates of HIV infection and syphilis and inventory of knowledge attitudes and risk practices related to sexually transmitted infections among drug users in 10 Brazilian municipalities. Technical Report Presented to the Department of STD, AIDS and Viral Hepatitis.

7. Department of Sexually Transmitted Infections, AIDS and Viral Hepatitis, Secretariat of Health Surveillance, Brazil Ministry of Health (2014) Epidemiological Bulletin HIVIAIDS.

8. Sabido M, Kerr LR, Mota RS, Benzaken AS, de A Pinho A, et al. (2015) Sexual violence against men who have sex with men in Brazil: $A$ respondent-driven sampling survey. AIDS Behav 19: 1630-1641.

9. Ferrer L, Furegato M, Foschia JP, Folch C, González V, et al. (2015) Undiagnosed HIV infection in a population of MSM from six European cities: Results from the Sialon project. Eur J Public Health 25: 494-500.

10. Bekker LG, Johnson L, Cowan F, Overs C, Besada D, et al. (2015) Combination HIV prevention for female sex workers: What is the evidence? Lancet 385: 72-87.

11. Khumalo-Sakutukwa G (2008) Project Accept (HPTN 043): A communitybased intervention to reduce HIV incidence in populations at risk for HIV in sub-Saharan Africa and Thailand. J Acquir Immune Defic Syndr 49: 422-431.

12. Sharma M, Ying R, Tarr G, Barnabas R (2013) Systematic review and metaanalysis of community and facility-based HIV testing to address linkage to care gaps in sub-Saharan Africa. Nature 528: S77-S85.

13. Krause J, Subklew-Sehume F, Kenyon C, Colebunders R (2013) Acceptability of HIV self-testing: A systematic literature review. BMC Public Health 13: 735.

14. Lorenc T, Marrero-Guillamon I, Aggleton P, Cooper C, Llewellyn A, et al. (2011) Promoting the uptake of HIV testing among men who have sex with men: Systematic review of effectiveness and cost-effectiveness. Sex Transm Infect 87: $272-278$

15. Stromdahl S, Hickson F, Pharris A, Sabido M, Baral S, et al. (2015) A systematic review of evidence to inform HIV prevention interventions among men who have sex with men in Europe. Euro Surveill 20: 21096.
16. Luchters S, Chersich MF, Rinyiru A (2008) Impact of five years of peer-mediated interventions on sexual behavior and sexually transmitted infections among female sex workers in Mombasa, Kenya. BMC Public Health 8: 143.

17. Chang LW, Serwadda D, Quinn TC, Wawer MJ, Gray RH, et al. (2013) Combination implementation for HIV prevention: Moving from clinical trial evidence to population-level effects. Lancet Infect Dis 13: 65-76.

18. Pascom ARP, Habckost CB, Lobo TDM (2016) Expanding HIV testing among key populations in Brazil: A peer point-of-care testing project experience. Bull World Health Organ 94: 626-630.

19. Pascom AR, Dutra de Barros CH, Lobo TD, et al. (2016) Point-of-care HIV tests done by peers, Brazil. Bull World Health Organ 94: 626-630.

20. Cappello JM, Gunasekera A, Gunasekera D, Ippolito T (2013) A multicenter performance evaluation of the DPP((R)) HIV-1/2 assay for the detection of HIV antibodies in various HIV testing algorithms. J Clin Virol 58: e59-e64.

21. Operario D, Soma T, Underhill K (2008) Sex work and HIV status among transgender women: Systematic review and meta-analysis. J Acquir Immune Defic Syndr 48: 97-103.

22. Lobato MI, Koff WJ, Crestana T, Chaves C, Salvador J, et al. (2009) Using the Defensive Style Questionnaire to evaluate the impact of sex reassignment surgery on defensive mechanisms in transsexual patients. Rev Bras Psiquiatr 31: 303-306.

23. Stephens SC, Bernstein KT, Philip SS (2011) Male to female and female to male transgender persons have different sexual risk behaviors yet similar rates of STDs and HIV. AIDS Behav 15: 683-686.

24. Fernandez-Balbuena S, de la Fuente L, Hoyos J, Rosales-Statkus ME, Barrio G, et al. (2014) Highly visible street-based HIV rapid testing: is it an attractive option for a previously untested population? A cross-sectional study. Sex Transm Infect 90: 112-118.

25. Guenter D, Greer J, Barbara A, Robinson G, Roberts J, et al. (2008) Rapid point-of-care HIV testing in community-based anonymous testing program: A valuable alternative to conventional testing. AIDS Patient Care STDS 22: 195204

26. Margolis AD, Joseph $H$, Belcher L, Hirshfield S, Chiasson MA, et al. (2012) Never testing for HIV' among men who have sex with men recruited from a sexual networking website, United States. AIDS Behav 16: 23-29.

27. Thornton AC, Delpech V, Kall MM, Nardone A (2012) HIV testing in community settings in resource-rich countries: A systematic review of the evidence. HIV Med 13: 416-426.

28. Sullivan PS, Carballo-Dieguez A, Coates T, Goodreau SM, McGowan I, et al. (2012) Successes and challenges of HIV prevention in men who have sex with men. Lancet 380: 388-399.

29. Department of Sexually Transmitted Infections, AIDS and Viral Hepatitis Secretariat of Health Surveillance, Brazil Ministry of Health (2015) Global AIDS Response Progress Reporting Narrative Report 2014.

30. Department of Sexually Transmitted Infections, AIDS and Viral Hepatitis. Secretariat of Health Surveillance, Brazil Ministry of Health (2016) Oral PreExposure Prophylaxis of HIV Infection - PrEP in Brazil. 